$\underline{\text { Supporting Information }}$

\title{
Role and Effect of Anions in the Construction of Silver Complexes Based on a Pyridylimidazole Ligand with L-type Coordination Vectors and Their Photoluminescence Properties
}

\author{
Jieun Lee, ${ }^{\dagger}$ Youngjin Kang, ${ }^{* \dagger}$ Nam Sung Cho, ${ }^{\S}$ and Ki-Min Park ${ }^{*}, *$ \\ ${ }^{\dagger}$ Division of Science Education, Kangwon National University, Chuncheon 24341, South Korea \\ ${ }^{\S}$ Electronics and Telecommunications Research Institute (ETRI), 161 Gajeong-dong, Yuseong-gu, \\ Daejeon 305-350, Republic of Korea \\ ${ }^{\ddagger}$ Research Institute of Natural Science, Gyeongsang National University, Jinju 52828, South Korea \\ *Corresponding author: E-mail: kangy@kangwon.ac.kr.; Kmpark@gnu.ac.kr
}

\section{Table of Contents}

1. Figure S1. Emission photo in solid state. (Left to right: Complexes 2, 1 and 3)

2. Figure S2. ${ }^{1} \mathrm{H}$ NMR spectrum of free ligand in $\mathrm{CD}_{3} \mathrm{CN}$.

3. Table S1. Inter- and intra-molecular $\mathrm{C}-\mathrm{H} \cdots \mathrm{O}, \mathrm{C}-\mathrm{H} \cdots \mathrm{N}, \mathrm{C}-\mathrm{H} \cdots \pi$, and $\pi-\pi$ interactions for $\mathbf{1}[\AA$ and $\left.{ }^{\circ}\right]$.

4. Table S2. Inter- and intra-molecular $\mathrm{O}-\mathrm{H} \cdots \mathrm{O}, \mathrm{C}-\mathrm{H} \cdots \mathrm{O}, \mathrm{C}-\mathrm{H} \cdots \pi$, and $\pi-\pi$ interactions for $2[\AA$ and $\left.{ }^{\circ}\right]$.

5. Table S3. Inter- and intra-molecular $\mathrm{C}-\mathrm{H} \cdots \mathrm{O}, \mathrm{C}-\mathrm{H} \cdots \mathrm{N}, \mathrm{C}-\mathrm{H} \cdots \pi$, and $\pi \cdots \pi$ interactions for $\mathbf{3}$ [ $\AA$ and ${ }^{\circ}$ ] 


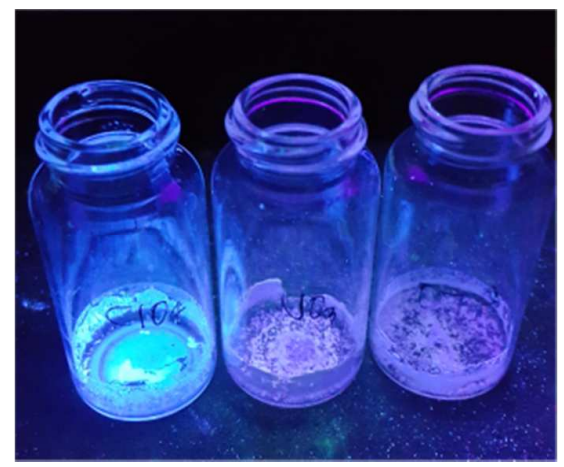

Figure S1. Emission photo in solid state. (Left to right: Complexes 2, 1 and 3)

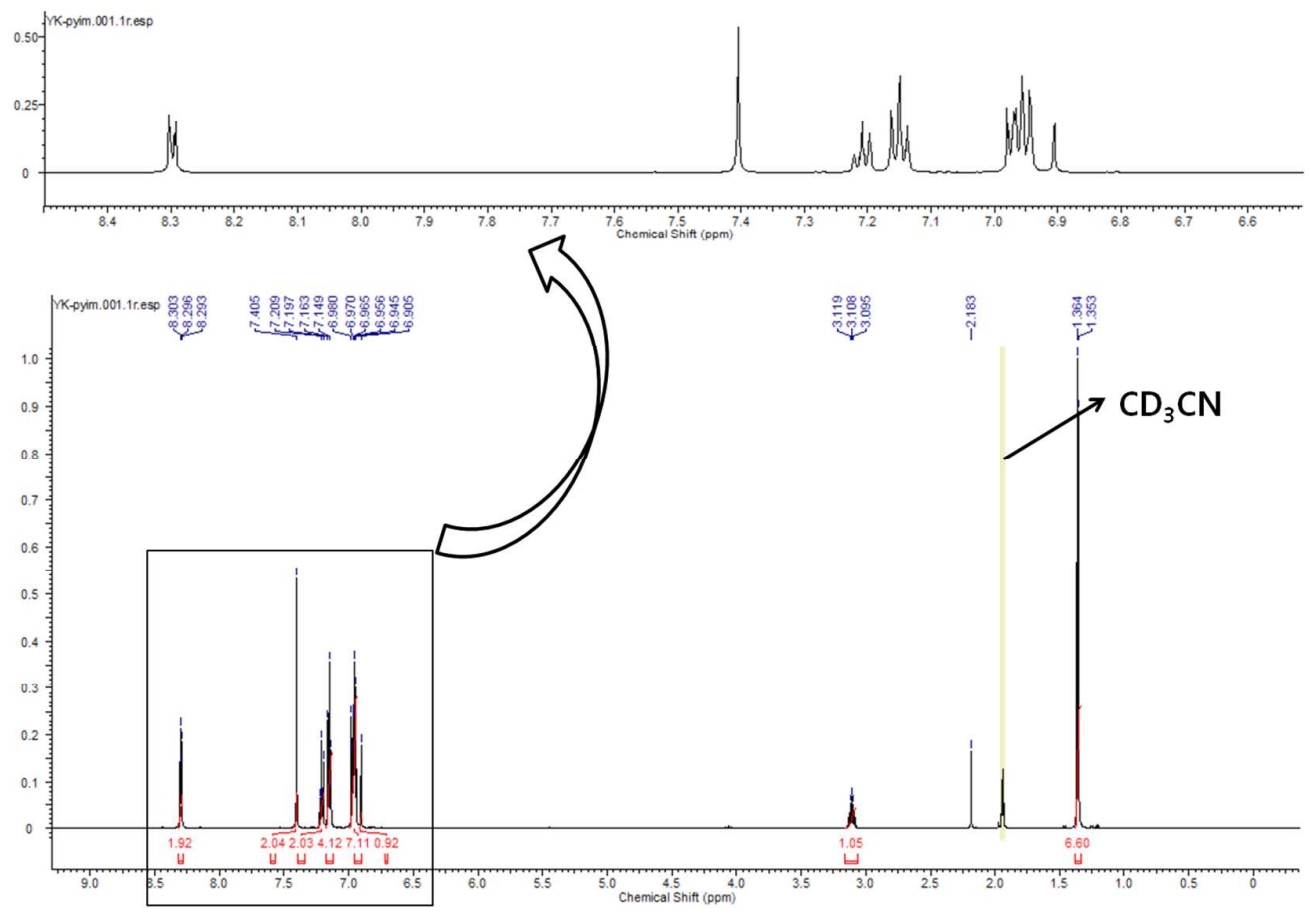

Figure S2. ${ }^{1} \mathrm{H}$ NMR spectrum of free ligand in $\mathrm{CD}_{3} \mathrm{CN}$. 
Table S1. Inter- and intra-molecular C-H $\cdots \mathrm{O}, \mathrm{C}-\mathrm{H} \cdots \mathrm{N}, \mathrm{C}-\mathrm{H} \cdots \pi$, and $\pi-\pi$ interactions for $\mathbf{1}\left[\AA\right.$ and $\left.^{\circ}\right]$. $\mathrm{Cg} 1, \mathrm{Cg} 2, \mathrm{Cg} 3, \mathrm{Cg} 4$, and $\mathrm{Cg} 5$ denote the centroids of aromatic rings $\mathrm{N} 1 / \mathrm{C} 1 / \mathrm{C} 2 / \mathrm{N} 2 / \mathrm{C} 3$, $\mathrm{N} 3 / \mathrm{C} 4 / \mathrm{C} 5 / \mathrm{C} 6 / \mathrm{C} 7 / \mathrm{C} 8, \mathrm{C} 9 / \mathrm{C} 10 / \mathrm{C} 11 / \mathrm{C} 12 / \mathrm{C} 13 / \mathrm{C} 14, \mathrm{C} 15 / \mathrm{C} 16 / \mathrm{C} 17 / \mathrm{C} 18 / \mathrm{C} 19 / \mathrm{C} 20$, and $\mathrm{C} 21 / \mathrm{C} 22 / \mathrm{C} 23 / \mathrm{C} 24 / \mathrm{C} 25 / \mathrm{C} 26$, respectively.

\begin{tabular}{lllll}
\hline $\mathrm{D}-\mathrm{H} \cdots \mathrm{A}$ & $\mathrm{d}(\mathrm{D}-\mathrm{H})$ & $\mathrm{d}(\mathrm{H} \cdots \mathrm{A})$ & $\mathrm{d}(\mathrm{D} \cdots \mathrm{A})$ & $<(\mathrm{DHA})$ \\
\hline $\mathrm{C} 6-\mathrm{H} 6 \cdots \mathrm{O} 1^{\mathrm{i}}$ & 0.95 & 2.45 & $3.293(2)$ & 148 \\
$\mathrm{C} 6-\mathrm{H} 6 \cdots \mathrm{O}^{\mathrm{i}}$ & 0.95 & 2.57 & $3.449(3)$ & 155 \\
$\mathrm{C} 13-\mathrm{H} 13 \cdots \mathrm{O} 2^{\mathrm{ii}}$ & 0.95 & 2.45 & $3.398(2)$ & 173 \\
$\mathrm{C} 16-\mathrm{H} 16 \cdots \mathrm{N} 2$ & 0.95 & 2.58 & $2.973(2)$ & 105 \\
$\mathrm{C} 29-\mathrm{H} 29 \mathrm{~B} \cdots \mathrm{O} 2^{\mathrm{ii}}$ & 0.98 & 2.49 & $3.471(2)$ & 175 \\
$\mathrm{C} 16-\mathrm{H} 16 \cdots \mathrm{Cg} 1$ & 0.95 & 2.84 & $3.321(2)$ & 112 \\
$\mathrm{C} 22-\mathrm{H} 22 \cdots \mathrm{Cg} 1$ & 0.95 & 2.92 & $3.3332(19)$ & 107 \\
$\mathrm{C} 22-\mathrm{H} 22 \cdots \mathrm{Cg} 3^{\mathrm{iii}}$ & 0.95 & 2.88 & $3.4863(18)$ & 123 \\
$\mathrm{Cg} 2 \cdots \mathrm{Cg} 2^{\mathrm{iv}}$ & & & $3.7744(10)$ & \\
$\mathrm{Cg} 2 \cdots \mathrm{Cg} 5$ & & & $3.8502(9)$ & \\
$\mathrm{Cg} 4 \cdots \mathrm{Cg} 4{ }^{\mathrm{v}}$ & & & $3.6693(10)$ & \\
\hline
\end{tabular}

Symmetry transformations used to generate equivalent atoms: (i) $x+1, y, z$; (ii) $x, y-1, z$; (iii) $-x,-y,-z$; (iv) $-x+1,-y+1,-z+1$; (v) $-x,-y,-z+1$. 
Table S2. Inter- and intra-molecular O-H $\cdots \mathrm{O}, \mathrm{C}-\mathrm{H} \cdots \mathrm{O}, \mathrm{C}-\mathrm{H} \cdots \pi$, and $\pi-\pi$ interactions for 2 [ $\AA$ and ${ }^{\circ}$ ]. $\mathrm{Cg} 1, \mathrm{Cg} 2, \mathrm{Cg} 3, \mathrm{Cg} 4$, and $\mathrm{Cg} 5$ denote the centroids of aromatic rings $\mathrm{N} 4 / \mathrm{C} 30 / \mathrm{C} 31 / \mathrm{N} 5 / \mathrm{C} 32$, $\mathrm{N} 3 / \mathrm{C} 4 / \mathrm{C} 5 / \mathrm{C} 6 / \mathrm{C} 7 / \mathrm{C} 8, \mathrm{~N} 6 / \mathrm{C} 33 / \mathrm{C} 34 / \mathrm{C} 35 / \mathrm{C} 36 / \mathrm{C} 37, \mathrm{C} 21 / \mathrm{C} 22 / \mathrm{C} 23 / \mathrm{C} 24 / \mathrm{C} 25 / \mathrm{C} 26$, and C50/C51/C52/C53/C54/C55, respectively.

\begin{tabular}{lllll}
\hline $\mathrm{D}-\mathrm{H} \cdots \mathrm{A}$ & $\mathrm{d}(\mathrm{D}-\mathrm{H})$ & $\mathrm{d}(\mathrm{H} \cdots \mathrm{A})$ & $\mathrm{d}(\mathrm{D} \cdots \mathrm{A})$ & $<(\mathrm{DHA})$ \\
\hline $\mathrm{O} 6-\mathrm{H} 6 \mathrm{~A} \cdots \mathrm{O} 2 \mathrm{WA}$ & 0.84 & 1.97 & $2.694(10)$ & 144 \\
$\mathrm{C} 13-\mathrm{H} 13 \cdots \mathrm{O} 2 \mathrm{WA}^{\mathrm{i}}$ & 0.95 & 2.44 & $3.383(8)$ & 173 \\
$\mathrm{C} 31-\mathrm{H} 31 \cdots \mathrm{O} 6{ }^{\mathrm{ii}}$ & 0.95 & 2.43 & $3.326(5)$ & 158 \\
$\mathrm{C} 51-\mathrm{H} 51 \cdots \mathrm{Cg} 1$ & 0.95 & 2.95 & $3.380(3)$ & 109 \\
$\mathrm{Cg} 2 \cdots \mathrm{Cg}{ }^{\mathrm{i}}$ & & & $3.9671(12)$ & \\
$\mathrm{Cg} 2 \cdots \mathrm{Cg} 4$ & & $3.8209(14)$ & \\
$\mathrm{Cg} 3 \cdots \mathrm{Cg} 3{ }^{\text {iii }}$ & & $3.9493(13)$ & \\
$\mathrm{Cg} 3 \cdots \mathrm{Cg} 5$ & & $3.7436(13)$ & \\
$\mathrm{Cg} 5 \cdots \mathrm{Cg} 5{ }^{\text {ii }}$ & & $3.7705(15)$ & \\
\hline
\end{tabular}

Symmetry transformations used to generate equivalent atoms: (i) $-x+1,-y+1,-z$; (ii) $-x+2,-y,-z+1$; (iii) $-x+2,-y+1,-z+1$. 
Table S3. Inter- and intra-molecular C-H $\cdots \mathrm{O}, \mathrm{C}-\mathrm{H} \cdots \mathrm{N}, \mathrm{C}-\mathrm{H} \cdots \pi$, and $\pi \cdots \pi$ interactions for 3 [ $\AA$ and ${ }^{\circ}$. $\mathrm{Cg} 1, \mathrm{Cg} 2, \mathrm{Cg} 3, \mathrm{Cg} 4, \mathrm{Cg} 5$, and $\mathrm{Cg} 6$ denote the centroids of aromatic rings $\mathrm{N} 1 / \mathrm{C} 1 / \mathrm{C} 2 / \mathrm{N} 2 / \mathrm{C} 3$, $\mathrm{N} 4 / \mathrm{C} 30 / \mathrm{C} 31 / \mathrm{N} 5 / \mathrm{C} 32, \mathrm{~N} 4 / \mathrm{C} 4 / \mathrm{C} 5 / \mathrm{C} 6 / \mathrm{C} 7 / \mathrm{C} 8, \mathrm{C} 9 / \mathrm{C} 10 / \mathrm{C} 11 / \mathrm{C} 12 / \mathrm{C} 13 / \mathrm{C} 14$, $\mathrm{C} 15 / \mathrm{C} 16 / \mathrm{C} 17 / \mathrm{C} 18 / \mathrm{C} 19 / \mathrm{C} 20$, and C21/C22/C23/C24/C25/C26, respectively.

\begin{tabular}{|c|c|c|c|c|}
\hline $\mathrm{D}-\mathrm{H} \cdots \mathrm{A}$ & $\mathrm{d}(\mathrm{D}-\mathrm{H})$ & $\mathrm{d}(\mathrm{H} \cdots \mathrm{A})$ & $\mathrm{d}(\mathrm{D} \cdots \mathrm{A})$ & $<(\mathrm{DHA})$ \\
\hline $\mathrm{C} 7-\mathrm{H}^{\prime} \cdots \mathrm{O}^{\prime}$ & 0.95 & 2.28 & $3.194(7)$ & 161 \\
\hline $\mathrm{C} 23-\mathrm{H} 23 \cdots \mathrm{F}^{\mathrm{i}}$ & 0.95 & 2.47 & $3.160(9)$ & 129 \\
\hline $\mathrm{C} 26-\mathrm{H} 26 \cdots \mathrm{O} 3^{\mathrm{ii}}$ & 0.95 & 2.53 & $3.401(4)$ & 153 \\
\hline $\mathrm{C} 30-\mathrm{H} 30 \cdots \mathrm{O} 2$ & 0.95 & 2.58 & $3.451(4)$ & 152 \\
\hline $\mathrm{C} 31-\mathrm{H} 31 \cdots \mathrm{O} 5^{\mathrm{iii}}$ & 0.95 & 2.38 & $3.313(8)$ & 168 \\
\hline $\mathrm{C} 31-\mathrm{H} 31 \cdots \mathrm{O}^{\text {,iii }}$ & 0.95 & 2.31 & $3.184(10)$ & 153 \\
\hline $\mathrm{C} 36-\mathrm{H} 36 \cdots \mathrm{O} 4^{\mathrm{iv}}$ & 0.95 & 2.44 & $2.960(7)$ & 114 \\
\hline $\mathrm{C} 36-\mathrm{H} 36 \cdots \mathrm{O} 6^{\mathrm{iv}}$ & 0.95 & 2.42 & $3.243(10)$ & 144 \\
\hline $\mathrm{C} 45-\mathrm{H} 45 \cdots \mathrm{O} 5^{\mathrm{iii}}$ & 0.95 & 2.49 & $3.347(8)$ & 150 \\
\hline $\mathrm{C} 45-\mathrm{H} 45 \cdots \mathrm{O} 5^{\text {,iii }}$ & 0.95 & 2.53 & $3.395(10)$ & 151 \\
\hline $\mathrm{C} 49-\mathrm{H} 49 \cdots \mathrm{F}^{\mathrm{iv}}$ & 0.95 & 2.36 & $3.251(9)$ & 156 \\
\hline $\mathrm{C} 51-\mathrm{H} 51 \cdots \mathrm{O}^{\mathrm{v}}$ & 0.95 & 2.49 & $3.381(4)$ & 157 \\
\hline $\mathrm{C} 55-\mathrm{H} 55 \cdots \mathrm{F}^{\mathrm{iiii}}$ & 0.95 & 2.48 & $3.218(11)$ & 134 \\
\hline $\mathrm{C} 55-\mathrm{H} 55 \cdots \mathrm{O}^{\mathrm{iii}}$ & 0.95 & 2.44 & $3.338(10)$ & 158 \\
\hline C58-H58A..F2 ${ }^{\mathrm{v}}$ & 0.98 & 2.39 & $3.252(5)$ & 146 \\
\hline $\mathrm{C} 16-\mathrm{H} 16 \cdots \mathrm{Cg} 1$ & 0.95 & 2.93 & $3.303(3)$ & 105 \\
\hline $\mathrm{C} 22-\mathrm{H} 22 \cdots \mathrm{Cg} 1$ & 0.95 & 2.94 & $3.392(3)$ & 110 \\
\hline $\mathrm{C} 52-\mathrm{H} 52 \cdots \mathrm{Cg} 2^{\mathrm{vi}}$ & 0.95 & 2.96 & $3.791(4)$ & 147 \\
\hline $\mathrm{C} 58-\mathrm{H} 58 \mathrm{C} \cdots \mathrm{Cg} 4^{\mathrm{vii}}$ & 0.98 & 2.64 & $3.493(5)$ & 144 \\
\hline $\mathrm{Cg} 3 \cdots \mathrm{Cg} 6$ & & & $3.920(2)$ & \\
\hline $\mathrm{Cg} 5 \cdots \mathrm{Cg} 5^{\text {viii }}$ & & & $3.807(2)$ & \\
\hline
\end{tabular}

Symmetry transformations used to generate equivalent atoms: (i) $x, y+1, z$; (ii) $x,-y+3 / 2, z-1 / 2$; (iii) $x+1, y+1 / 2,-z+1 / 2$; (iv) $-x+1,-y+1,-z$; (v) $-x+1, y-1 / 2,-z+1 / 2$; (vi) $-x+1, y-1 / 2,-z+1 / 2$; (vii) $x+1,-$ $y+3 / 2, z+1 / 2$; (viii) $-x,-y+1,-z$. 